\title{
Improvement of motor evoked potentials monitoring is required during thoracic or thoracoabdominal aortic aneurysm surgery under hypothermic cardiopulmonary bypass
}

\author{
Masahiko Kawaguchi • Mikito Kawamata • \\ Yoshitsugu Yamada
}

Received: 10 January 2012 / Accepted: 15 January 2012/Published online: 23 February 2012

(C) Japanese Society of Anesthesiologists 2012

Keywords Motor evoked potential - Thoracoabdominal aortic aneurysm · Hypothermia

Monitoring of motor evoked potentials (MEPs) can provide information about the functional integrity of a motor pathway and, therefore, has been widely used during surgery in which a motor pathway is at risk of injury, including craniotomy, spine/spinal surgery, and thoracic or thoracoabdominal aortic aneurysm (TAA or TAAA) surgery $[1,2]$. Because myogenic MEPs are very sensitive to suppression by anesthetic agents and neuromuscular blockade, careful attention should be paid to anesthetic management during monitoring of MEPs. In general, total intravenous anesthesia with propofol and opioids is used, although ketamine-based anesthesia may be applied in cases in which recording of myogenic MEPs is not reliable because of suppression by anesthetic agents or influence by preoperative motor dysfunction.

Of the cases in which MEPs monitoring is required, TAA/TAAA surgery is the most challenging for reliable

This comment refers to the article available at doi: 10.1007/s00540-011-1313-2.

M. Kawaguchi $(\bowtie)$

Department of Anesthesiology, Nara Medical University, 840 Shijo-cho, Kashihara, Nara 634-8522, Japan

e-mail: drjkawa@naramed-u.ac.jp

M. Kawamata

Department of Anesthesiology and Resuscitology,

Shinshu University School of Medicine, Matsumoto, Japan

Y. Yamada

Department of Anesthesiology, Tokyo University Graduate

School of Medicine, Tokyo, Japan
MEP monitoring. In addition to the effects of anesthetics, a variety of factors can affect the recording of MEPs, including hypothermia, aortic clamp, and induction of cardiopulmonary bypass (CPB). Hypothermia may have direct effects on the MEP itself and also indirect effects on MEPs by changing the pharmacokinetics and pharmacodynamics of anesthetic agents, modified by the effects of aortic clamp and the induction of CPB. Leslie et al. [3] demonstrated that a temperature reduction of $3^{\circ} \mathrm{C}$ increased blood propofol concentration by $30^{\circ} \mathrm{C}$ during a constant rate infusion. Kakinohana et al. [4] reported that plasma propofol concentration increased and bispectral index values decreased rapidly after aortic cross-clamping in thoracic aortic aneurysm repair surgery during propofol anesthesia. Yoshitani et al. [5] demonstrated that, in patients receiving propofol infusion at a rate of 5 or $6 \mathrm{mg} / \mathrm{kg} / \mathrm{h}$, plasma propofol concentration increased and burst suppression rate on electroencephalogram increased after the induction of normothermic CPB, compared with those values before CPB. These findings suggest that interpretation of MEP changes could be difficult because anesthetic level can easily fluctuate during TAAA surgery with hypothermic CPB.

Data regarding the effects of hypothermia on myogenic MEPs have been limited to animal experiments. Meylaerts et al. [6] investigated the effects of regional spinal cord hypothermia on myogenic MEPs induced by transcranial train-pulse stimulation in pigs anesthetized with ketamine, sufentanil, clonidine, and nitrous oxide. They demonstrated that progressive cooling resulted in an increase of MEPs at $28^{\circ}-30^{\circ} \mathrm{C}$ and was followed by a progressive decrease in MEP amplitude. MEP amplitude decreased below $25 \%$ at approximately $14^{\circ} \mathrm{C}$. Sakamoto et al. [7] also investigated the effects of systemic hypothermia on myogenic MEPs induced by a single pulse and a train of pulses under 
propofol, ketamine, and fentanyl anesthesia in rabbits. The results indicated that MEP amplitude induced by singlepulse stimulation was significantly reduced with a reduction of core temperature to $28^{\circ} \mathrm{C}$ compared with the control value at $38^{\circ} \mathrm{C}$, whereas MEP amplitude induced by train-pulse stimulation did not change significantly during the cooling to $28^{\circ} \mathrm{C}$. In both experiments, MEP latency was increased linearly with a decrease in spinal or core temperature.

There had been no human study to investigate extensively the effects of deep hypothermia on myogenic MEPs in patients undergoing TAA/TAAA surgery under moderate to deep hypothermia. The recent study by Shinzawa et al. [8] demonstrated interesting findings of myogenic MEPs changes during deep hypothermic circulatory arrest (DHCA) in TAA/TAAA surgery under propofol and remifentanil anesthesia. In the cooling phase, MEP amplitude decreased linearly as nasopharyngeal temperature (NPT) decreased. MEPs from both hand and leg muscles disappeared at approximately $16^{\circ} \mathrm{C}$ in 10 of 15 patients and still remained elicited in 5 patients. In the rewarming phase, MEPs from hand muscle had a peak in amplitude at $25^{\circ} \mathrm{C}$ with an amplitude of $58.5 \%$ of the controls. However, MEPs amplitude began to decrease after the peak in spite of an increase of body temperature and was $15.6 \%$ of the controls at $36^{\circ} \mathrm{C}$. As indicated in the animal studies, MEP monitoring seems to be feasible, at least above $25^{\circ} \mathrm{C}$, in patients undergoing TAA/TAAA surgery, although there may be wide variability in amplitudes between patients.

The report by Shinzawa et al. raised several points, one of which is reliability of the cutoff value for the alert of MEP changes. Cutoff values for MEP amplitudes have been debated based on only data in a small number of patients. Several authors may have used a cutoff point for the alarm at 75\% decrease of baseline MEP [9]. However, based on the data by Shinzawa et al., MEP amplitudes can easily fluctuate during the rewarming phase. For example, during rewarming from $25^{\circ}$ to $37^{\circ} \mathrm{C}$, MEP amplitude may decrease and reach the cutoff point for alarm, which may mislead surgeons and anesthesiologists to do unnecessary interventions to improve spinal cord blood supply. In addition, a wide variability in MEP amplitudes during moderate and deep hypothermic CPB within the subject may also make the assessment of MEPs results difficult.

The other point is the ability of myogenic MEPs to predict postoperative motor dysfunction in patients undergoing TAA/TAAA surgery with hypothermic CPB. In cases undergoing TAA/TAAA surgery with moderate hypothermic $\mathrm{CPB}$, reported sensitivity and specificity to predict postoperative paraplegia have not been satisfactory, ranging from $62 \%$ to $100 \%$ for sensitivity and from $39 \%$ to $100 \%$ for specificity [9]. In the reports by Shinzawa et al., MEPs in the leg did not recover in the rewarming phase in
8 of 15 patients, although there were no cases with postoperative motor dysfunction. Deep hypothermia itself might have provided neuroprotective effects on ischemic spinal cord. However, the benefit to perform MEP monitoring is unclear, because it is difficult to decide whether further interventions would be required based on the results of MEPs during the main surgical procedure.

Finally, Shinzawa et al. emphasized the importance of the location of temperature recording. They demonstrated a discrepancy between the core and peripheral temperature in TAA/TAAA surgery. Especially, in the rewarming phase, there was a delay in an increase of leg temperature compared with NPT and plantar temperature. Even at the end of $\mathrm{CPB}$, leg temperature values were lower compared with those of NPT and plantar temperature. These findings suggest that the location of temperature monitoring should be standardized and MEPs from the upper limb may not be accurate as a control for MEPs from the lower limb because the temperature of upper and lower limbs may differ.

These findings suggest that further research is needed regarding anesthetic management to provide the conditions in which appropriate MEP responses can be recorded, even during hypothermic CPB. Based on MEP data under conditions of sophisticated anesthetic management, the cutoff point for the alarm and the ability of MEPs to predict postoperative paraplegia should be evaluated in a large number of patients undergoing TAA/TAAA surgery in a prospective manner.

\section{References}

1. Jacobs MJ, Mess W, Mochtar B, Nijenhuis RJ, Statius van Eps RG, Schurink GW. The value of motor evoked potentials in reducing paraplegia during thoracoabdominal aneurysm repair. J Vasc Surg. 2006;43(2):239-46.

2. Kawaguchi M, Furuya $H$. Intraoperative spinal cord monitoring of motor function with myogenic motor evoked potentials: a consideration in anesthesia. J Anesth. 2004;18(1):18-28.

3. Leslie K, Bjorksten AR, Ugoni A, Mitchell P. Mild core hypothermia and anesthetic requirement for loss of responsiveness during propofol anesthesia for craniotomy. Anesth Analg. 2002; 94(5):1298-303.

4. Kakinohana M, Nakamura S, Fuchigami T, Miyata Y, Sugahara K. Influence of the descending thoracic aortic cross clamping on bispectral index value and plasma propofol concentration in humans. Anesthesiology. 2006;104(5):939-43.

5. Yoshitani K, Kawaguchi M, Takahashi M, Kitaguchi K, Furuya H. Plasma propofol concentration and EEG burst suppression ratio during normothermic cardiopulmonary bypass. Br J Anaesth. 2003; 90(2):122-6.

6. Meylaerts SA, De Haan P, Kalkman CJ, Lips J, De Mol BA, Jacobs MJ. The influence of regional spinal cord hypothermia on transcranial myogenic motor-evoked potential monitoring and the efficacy of spinal cord ischemia detection. $\mathbf{J}$ Thorac Cardiovasc Surg. 1999;118(6):1038-45. 
7. Sakamoto T, Kawaguchi M, Kakimoto M, Inoue S, Takahashi M, Furuya $\mathrm{H}$. The effect of hypothermia on myogenic motor-evoked potentials to electrical stimulation with a single pulse and a train of pulses under propofol/ketamine/fentanyl anesthesia in rabbits. Anesth Analg. 2003;96(6):1692-7.

8. Shinzawa M, Yoshitani K, Minatoya K, Irie T, Ogino H, Ohnishi $\mathrm{Y}$. Changes of motor evoked potentials during descending thoracic and thoracoabdominal aortic surgery with deep hypothermic circulatory arrest. J Anesth 2012 [Epub ahead of print]

9. Horiuchi T, Kawaguchi M, Inoue S, Hayashi H, Abe R, Tabayashi $\mathrm{N}$, Taniguchi S, Furuya H. Assessment of intraoperative motor evoked potentials for predicting postoperative paraplegia in thoracic and thoracoabdominal aortic aneurysm repair. J Anesth. 2011;25(1):18-28. 